\title{
Original
}

\section{The Prognostic Significance of Expression of CA125 and Proliferation Markers and Architectural Type in Ovarian Clear Cell Adenocarcinoma}

\author{
Tsuyoshi Okuda ${ }^{1,4)}$, Hiroshi Saito", Akihiko Sekizawa ${ }^{1)}$, Tatsuya Akamatsu ${ }^{1)}$, \\ Akira Suzuki ${ }^{1)}$, Atsushi Shimizu ${ }^{1)}$, Miki Kushima ${ }^{2)}$, Hidekazu OTA ${ }^{3)}$ \\ and Takumi YanalHara ${ }^{1)}$
}

\begin{abstract}
The prognosis of ovarian clear cell adenocarcinoma remains poor, even in the early stages. Thus, great efforts are being made to improve the treatment modalities of this disease, with chemotherapy in particular being in a period of dynamic development. To date, no universal criteria of histological grade or effective prognostic factors have been established for ovarian clear cell adenocarcinoma. The purpose of the present study was to investigate the prognostic significance of histological architectural subtype, immunohistochemical staining with CA125 and proliferation factors in ovarian clear cell adenocarcinoma tissues. Tumors obtained from 24 patients with ovarian clear cell adenocarcinoma (Stage I-IV) were examined for architectural subtype and immunohistochemical expression of CA125, PCNA, Ki-67, and topoisomerase II. Architectural subtype was classified into three types (papillary, solid, and tubulocystic/glandular). The expression of CA125, PCNA, Ki-67, and topoisomerase II were compared with clinical features of the study population and overall survival. Survival curves were constructed according to the Kaplan-Meier method. The PCNA, Ki-67 and topoisomerase II labeling indices in the tumors were $44.3 \pm 14.7 \%, 14.0 \pm 5.0 \%$ and $13.9 \pm$ $7.7 \%$ (mean \pm S.D.), respectively. Strong expression of CA125 was observed in $41.7 \%$ of the tumors. This expression was not associated with FIGO stage or architectural subtype. Patients with papillary type carcinoma had a lower probability of survival than those with the solid type. Patients with strong expression of CA125 demonstrated a higher probability of survival than those with weak expression. These results suggest that low immunohistochemical expression of CA125 and papillary subtype in ovarian clear cell adenocarcinoma are associated with a poor prognosis.
\end{abstract}

Key words : ovarian clear cell adenocarcinoma, CA125, proliferating factors, immunohistochemical staining, architectural type

\footnotetext{
1) Department of Obstetrics and Gynecology, Showa University School of Medicine, 1-5-8 Hatanodai, Shinagawa-ku, Tokyo 142-8666, Japan.

2) Department of Hospital Pathology, Showa University School of Medicine.

3) 2nd Department of Pathology, Showa University School of Medicine.

4) Department of Obstetrics and Gynecology, Showa University School of Medicine.
} 


\section{Introduction}

The incidence of ovarian clear cell adenocarcinoma (OCCA) has been increasing but the origin of the disease remains unclear. Endometriosis has been reported in association with certain epithelial ovarian tumors, although in itself it is a benign condition. Scully and Barlow $^{1)}$ reported a close association between endometriosis and OCCA or endometrioid carcinoma of the ovary, and several authors have demonstrated the coexistence of endometriosis in patients with $\mathrm{OCCA}^{2-4)}$.

Histological tumor staging has been developed in the current International Federation of Gynecology and Obstetrics (FIGO) staging classification ${ }^{5)}$, but not for OCCA grade, despite a growing awareness of its prognostic importance regardless of stage and histology. Cancer antigen 125 (CA125), a glycoprotein expressed on the surface of most ovarian epithelial carcinomas $^{6}$ and serum CA125 levels were initially described as markers of epitherial ovarian carcinoma ${ }^{7)}$. Serum levels of CA125 increases with progression of the endometriotic lesion. Positive and negative cells are intimately intermixed within CA125-positive tumors, including endometriosis, demonstrating tumor cell heterogeneity ${ }^{6,8)}$. The prognostic significance of the proportion of cells expressing CA125 within the tumor for OCCA patients has yet to be examined. Shimizu et al. ${ }^{9)}$ reported that survival rate is related to architectural grade, nuclear pleomorphism and mitotic figures in ovarian carcinoma except OCCA. In the present study, to evaluate the prognostic significance of various factors in OCCA, we classified the architectural types evident in 24 patients and examined expression of CA125 by immunohistochemistry. We also performed immunohistochemical staining of the cell proliferation markers proliferating cell nuclear antigen (PCNA), Ki-67, and topoisomerase II. We further analysed the clinicopathological variables in relation to architectural type, and expression of CA125 and proliferating factors.

\section{Patients and Methods}

\section{Patients}

A total of 24 Japanese patients (median age 51 years, range; 29-72 years) with pure OCCA who underwent primary surgery at Showa University Hospital, Japan, between 1990 and 1998 was examined. All histologic samples were taken at laparotomy before chemotherapy was initiated. Informed consent was obtained from all patients.

Clinical staging was determined according to the FIGO staging system ${ }^{5)}$. The proportion of patients in each surgical stage was as follows: Stage I, 13 (54.2\%); Stage II, 1 (4.2\%); Stage III, $8(33.3 \%)$; and Stage IV, $2(8.3 \%)$. Optimal cytoreduction was achieved in all 24 patients. After primary cytoreductive surgery, all patients recieved platinum-based chemotherapy. Follow-up information on surviving patients was obtained through to September 30th, 1999, via direct contact with the patients, their families, or their physicians.

\section{Histology}

Histopathologic diagnosis was based on World Health Organaization (WHO) criteria ${ }^{10)}$. The tumors were classified into three architectural subtypes (papillary, solid, and tubulocystic/glandular). 
Table 1. Primary immunohistochemical antibodies (mouse monoclonal antibodies) used in the present study

\begin{tabular}{llc}
\hline \multicolumn{1}{c}{ Detected antigen } & \multicolumn{1}{c}{ Source } & Dilution \\
\hline CA125 & DAKO & $1: 20$ \\
PCNA & NOVOCASTRA & $1: 200$ \\
Ki-67 & Immunotech & $1: 50$ \\
topoisomerase II & DAKO & $1: 50$ \\
\hline
\end{tabular}

\section{Immunohistochemistry}

Formalin-fixed, paraffin-embedded tissue was cut into 5- $\mu \mathrm{m}$ sections. Sections were then deparaffinized in xylene (twice for 5 minutes), fixed in a graded ethanol series for 3 minutes in each solution and washed for 5 minutes under a stream of water. Endogenous peroxidase activity was blocked using $3 \% \mathrm{H}_{2} \mathrm{O}_{2}$ for 20 minutes at room temperature (RT). Sections were then washed 3 times for 5 minutes in phosphate-buffered saline (PBS) and then stained using the labelled streptavidin biotin (LSAB) method. Briefly, sections were incubated at $4{ }^{\circ} \mathrm{C}$ overnight with one of the primary antibodies (to CA125, PCNA, Ki-67 or topoisomerase II, Table 1), followed by washing as before and incubation in mouse biotinylated anti-mouse IgG (DAKO, Denmark) (1:500) at RT for 30 minutes. After washing as before, the slides were incubated with streptavidin-conjugated horseradish peroxidase (DAKO, Denmark) for 30 minutes at RT, washed, and stained with 3,3'diaminobenzidine (DAB, Wako, Japan) with $0.3 \% \quad \mathrm{H}_{2} \mathrm{O}_{2}$ for 5 minutes. Nuclei were counterstained with hematoxylin. Positive and negative controls of staining were performed and were included in each staining run. Staining was assessed using a consulting microscope (Model BX50; Olympus, Tokyo, Japan) by two of the authors (TO, MK), both of whom had no prior knowledge of the clinical or follow-up data of the patients.

In all positive sections, PCNA, Ki-67 and topoisomerase II staining was localized to the cell nucleus. Negative control sections, from which primary antibody had been omitted, were included in all staining runs. The percentage of stained tumor cells per 1,000 cells was used as the PCNA, Ki-67, and topoisomerase II labeling indices.

CA125 labeling was confined to the cytoplasmic membranes of tumor cells and was considered weak if less than $50 \%$ of the cancer cells were positive for CA125 and strong if $50 \%$ or more of the malignant cells were CA125-positive (Fig. 1). Immunohistochemical staining was assessed without prior knowledge of survival or other clinical data. Results were also analyzed in relation to clinicopathological variables.

\section{Statistics}

Statistical analysis was performed using Fisher's exact test ${ }^{11)}$. Survival curves were constructed according to the Kaplan-Meier method ${ }^{12)}$. Differences in survival were assessed using the generalized Wilcoxon test ${ }^{13)}$. Statistical significance was established at the $p<0.05$ level. Statistical analysis was performed using the Stat View J software package, Version 4.5.

\section{Results}

The median duration of follow-up for surviving patients was 28.4 months (range ; 1-71 

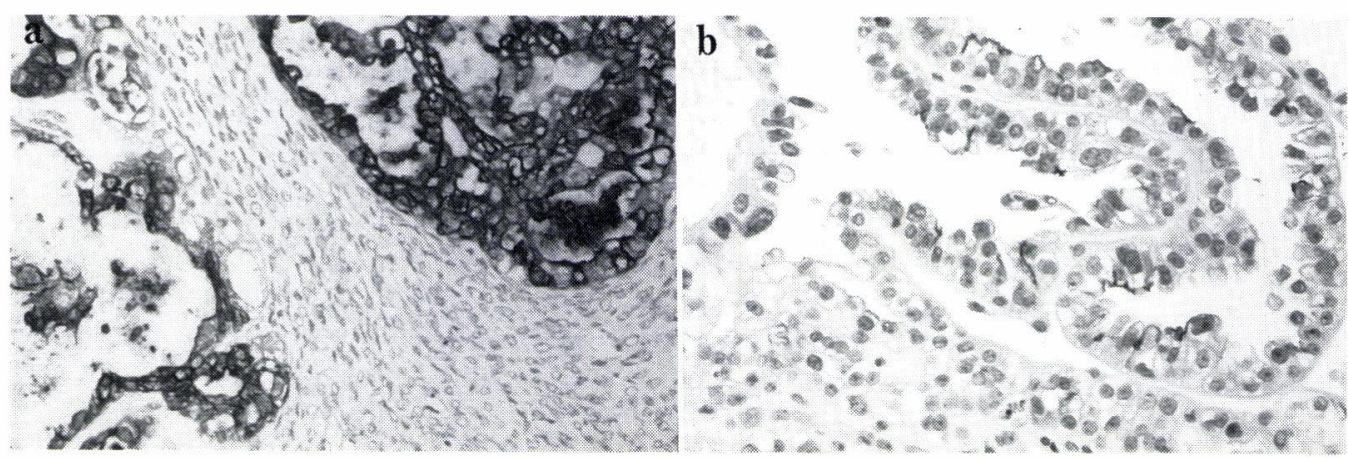

Fig. 1. Ovarian clear cell adenocarcinoma showing immunohistochemical staining of tumor cells with CA125. Note that CA125 staining is confined to the cytoplasmic membranes of tumor cells. a : Strong CA125 staining $(\times 200), b$ : Weak CA125 staining $(\times 200)$

months). The percentage of surviving patients followed for $\geqq 3$ years, $\geqq 2$ years, and 1.5 years was $37.5 \%, 58.3 \%$, and $62.5 \%$, respectively. At the time of the last follow-up, 10 of the 24 patients had died of ovarian carcinoma, 1 patient died without evidence of the disease and 13 patients were living with no evidence of the disease. Histological subtyping according to FIGO stage is shown in Table 2. Overall survival according to the different architectural subtypes is depicted in Fig. 2. The overall median survival of patients whose tumors were papillary, solid and tubulocystic/glandular type was 21.2, 44.6 and 29.0 months, respectively. The survival of patients with papillary-type tumors was significantly shorter than that of those with the solid type $(\mathbf{p}<0.01)$.

Immunoreactivity for PCNA, Ki-67, topoisomerase II and CA125 according to FIGO stage and pathological variables are shown in Tables 3 and 4 . The labelling indices of PCNA, Ki-67 and topoisomerase II, and expression of CA125, were not significantly related to FIGO stage. No significant association was observed between the expression of PCNA, Ki-67 or topoisomerase II and architectural type (Table 4, p $<0.05$ ). However, strong expression of CA125 was significantly lower in papillary type tumors (14.3\%) than in the solid $(83.3 \%)$ and tubulocystic/glandular $(75.0 \%)$ types. Although 17 of the 24 OCCA patients had coexistent endometriosis, this did not appear to influence the expression of PCNA, Ki-67, topoisomerase II or CA125.

Survival curves according to immunohistochemical CA125 expression are shown in Fig. 3. There was a significant difference in survival between the patients with strong expression of CA125 and those with weak expression $(\mathrm{p}<0.01)$.

\section{Discussion}

OCCA may manifest as one of a few histological types. Several studies have suggested the possibility of malignant transformation of endometriosis to $\mathrm{OCCA}^{2-4)}$. Although the prognosis for patients with OCCA remains poor, even in the early stages, no clear significant prognostic factor, after adjustment for more important prognostic factors such as stage and size of residual tumor after cytoreductive surgery, has ever been demonstrated. The grading of OCCAs is problematic and complicated by the multiplicity of histologic patterns found in OCCA patients ${ }^{9,14)}$. Kennedy et al. ${ }^{14)}$ reported no significant association between survival and age, history of endometriosis, gross appearance of tumor (solid or 
Table 2. Architectural type according to FIGO stage Sixty percent of the papillary type tumors were FIGO stage I

\begin{tabular}{lcccc}
\hline & \multicolumn{4}{c}{ FIGO Stage } \\
\cline { 2 - 5 } Architectural type & $\mathrm{I}$ & $\mathrm{I}$ & $\mathrm{II}$ & $\mathrm{IV}$ \\
& $(\mathrm{n}=13)$ & $(\mathrm{n}=1)$ & $(\mathrm{n}=8)$ & $(\mathrm{n}=2)$ \\
\hline papillary & 8 & 1 & 3 & 1 \\
solid & 3 & 0 & 3 & 1 \\
tubulocystic/glandular & 2 & 0 & 2 & 0 \\
\hline
\end{tabular}

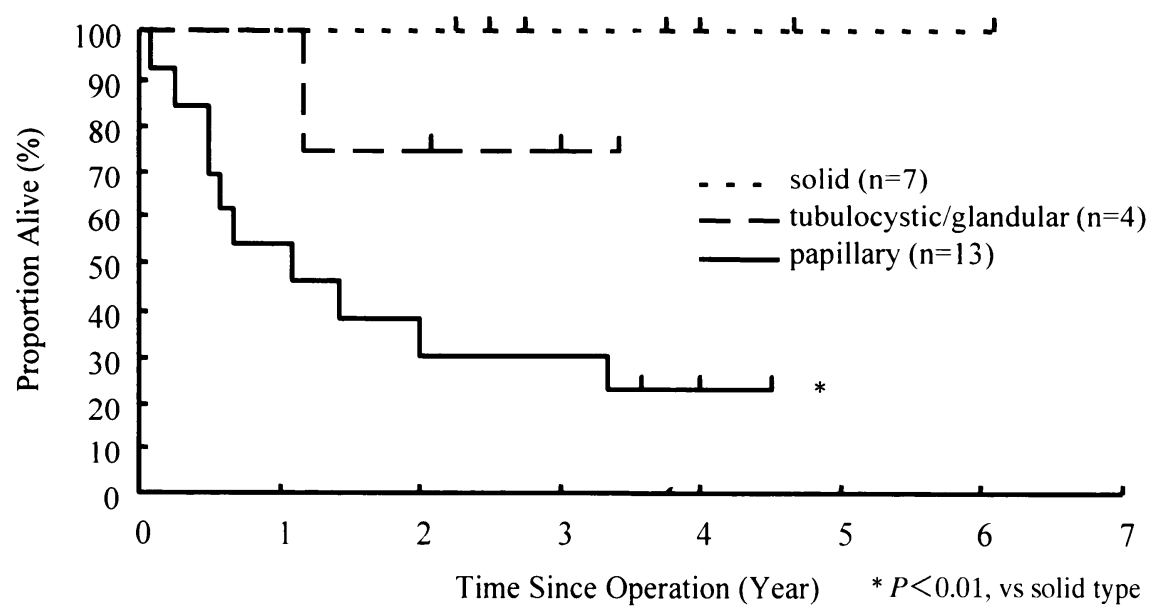

Fig. 2. Overall survival of patients in OCCA according to architectural type The survival of patients with papillary type tumor was significantly shorter than that of patients with solid type.

Table 3. The labelling indices of PCNA, Ki-67 and topoisomerase II, and expression of CA125 according to FIGO stage

\begin{tabular}{lcccc}
\hline & \multicolumn{4}{c}{ FIGO Stage } \\
\cline { 2 - 5 } & $\begin{array}{c}\text { I } \\
(\mathrm{n}=13)\end{array}$ & $\begin{array}{c}\mathrm{II} \\
(\mathrm{n}=1)\end{array}$ & $\begin{array}{c}\text { III } \\
(\mathrm{n}=8)\end{array}$ & $\begin{array}{c}\text { IV } \\
(\mathrm{n}=2)\end{array}$ \\
\hline PCNA labeling index & $40.8 \pm 15.7$ & 49.0 & $47.6 \pm 13.1$ & $56.0 \pm 6.0$ \\
Ki-67 labeling index & $13.4 \pm 5.2$ & 18.0 & $13.9 \pm 5.1$ & $12.5 \pm 02.5$ \\
Topoisomerase II labeling index & $11.9 \pm 6.8$ & 38.0 & $13.5 \pm 4.5$ & $12.5 \pm 2.5$ \\
CA125 strong/total (\%) & 38.5 & 0 & 50.0 & 50.0 \\
\hline
\end{tabular}

cystic), histologic pattern, relative percentage of cells with clear cytoplasma, or mitotic activity. In the present study, although $60 \%$ of the papillary type tumors were found in FIGO stage I, the overall survival of patients with the papillary type was significantly lower than that for those with the solid type (Fig. 2). Nakano et al. ${ }^{15)}$ examined the architectural pattern and overall survival for 31 cases of OCCA with results similar to those obtained in the present study. However, only 24 OCCA cases were examined here and additional cases 
Table 4. The labelling indices of PCNA, Ki-67 and topoisomerse II, and expression of CA125 according to pathological variables

\begin{tabular}{|c|c|c|c|c|c|}
\hline & \multicolumn{3}{|c|}{ Architectural type } & \multicolumn{2}{|c|}{ Endometriosis } \\
\hline & $\begin{array}{l}\text { papillary } \\
(n=13)\end{array}$ & $\begin{array}{c}\text { solid } \\
(n=7)\end{array}$ & $\begin{array}{l}\text { tubulocystic } \\
\text { /glandular } \\
(n=4)\end{array}$ & $\begin{array}{c}\text { with } \\
(\mathbf{n}=17)\end{array}$ & $\begin{array}{l}\text { without } \\
(\mathrm{n}=7)\end{array}$ \\
\hline abeling index & $42.6 \pm 12.0$ & $39.6 \pm 15.2$ & $46.0 \pm 19.5$ & $47.6 \pm 15.3$ & $36.1 \pm 9.3$ \\
\hline abeling index & $14.5 \pm 4.0$ & $12.0 \pm 6.3$ & $15.8 \pm 4.2$ & $14.0 \pm 5.0$ & $14.0 \pm 4.3$ \\
\hline amerase II labeling index & $14.1 \pm 9.1$ & $13.1 \pm 5.9$ & $14.8 \pm 4.3$ & $14.1 \pm 8.3$ & $13.6 \pm 5.7$ \\
\hline strong/total $(\%)$ & $14.3^{*}$ & 83.3 & 75.0 & 41.1 & 42.9 \\
\hline
\end{tabular}

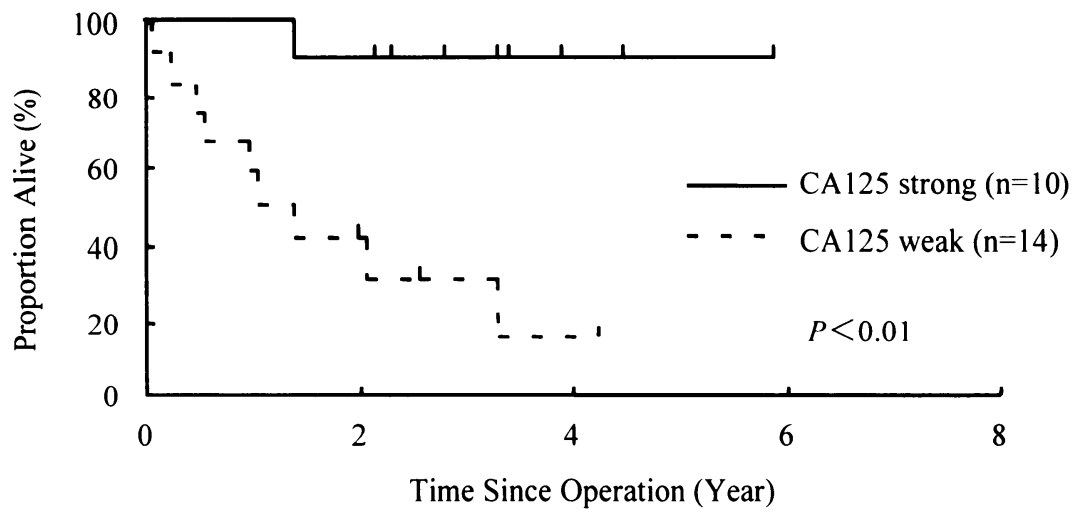

Fig. 3. Overall survival of patients in OCCA according to CA125 expression. The survival of patiernts with weak expression of CA125 was significantly shorter than that of patients with strong expression.

are required to determine the clinicopathological factors that have significant prognostic value.

PCNA is a co-enzyme of DNA polymerase- $\delta$, synthesized in the late G1 to $\mathrm{S}$ phase. The monoclonal antibody to $\mathrm{Ki}-67$ recognizes all nuclei in the cell-proliferating phase except G0. Both have been repeatedly been shown to correlate well with cell proliferation. DNA topoisomerase II plays an important catalytic role in regulating the topologic status of DNA. High expression of PCNA has been detected in $33 \%$ of ovarian adenocarcinomas ${ }^{16)}$. Furthermore, another study has shown an inverse correlation between PCNA expression and overall survival time in advanced ovarian carcinoma ${ }^{17)}$. These authors concluded that PCNA expression defines a good prognostic subgroup in patients with adequately debulked ovarian cancer. Several studies have shown that the PCNA index correlates with other means of assessing the proliferative activity of tumors, such as synthetic phase fraction analysis by DNA flow cytometry ${ }^{17,18)}$, the bromodeoxyuridine incorporation index ${ }^{19)}$, and the Ki-67 labeling index ${ }^{18-22)}$. In particular, the PCNA index shows a good correlation with the $\mathrm{Ki}-67$ (MIB-1) index that is widely used to estimate proliferative activity in formalin-fixed 
paraffin-embedded tissues ${ }^{20-22)}$. However, some studies have shown a lack of correlation between the PCNA and Ki-67 indices in breast and gastric carcinoma ${ }^{23-25)}$.

We examined the association between clinical variables and both architectural type and PCNA, Ki-67, topoisomerase II and CA125 expression to assess their potential prognostic significance. There were no significant differences between the labeling indices of the PCNA, Ki-67, and topoisomerase II proliferating factors, FIGO staging and architectural subtyping in the OCCA patients. Adachi et al. ${ }^{26)}$ described immunohistochemical staining of CA125 in ovarian adenocarcinoma, however they did not define the relationship between prognostic significance and CA125 expression in the tumor. To our knowledge, the present study is the first to demonstrate that the overall survival of OCCA patients with weak immunohistochemical CA125 staining in the tumor is significantly $(\mathrm{p}<0.01)$ shorter than that of those with strong CA125 staining. In addition, significantly fewer patients with papillary type OCCA had strong CA125 expression than patients with the other subtypes. In the present study, 17 of the 24 OCCA patients had endometriosis $(70.8 \%)$, although the expression of CA125 in the OCCA tissue was not influenced by endometriosis. Within CA125-positive tumors, positive and negative cells are found to be closely intermixed, demonstrating tumor cell heterogeneity. This heterogeneity of CA125 expression in ovarian tumor cells has important implications for the tumor response to systemic chemotherapy ${ }^{27)}$. Thus, one of the reasons for the poor responsiveness to chemotherapy of OCCA tumors may be a loss of CA125 expression.

In conclusion, OCCA patients with papillary type tumor and/or weak CA125 expression are considered to have a poor prognosis even in the early stage of this disease.

\section{References}

1) Scully RE and Barlow JF: Mesonephroma of ovary. Tumor of mullerian nature related the endometrioid carcinoma. Cancer 20 : 1405-1417 (1967)

2) Tohya T, Shibata S, Fujisaki $\mathbf{S}$ and Maeyama $\mathbf{M}$ : Clear cell carcinoma and endometrioid carcinoma arising in an endometriotic cyst of the ovary. Nippon Sanka Fuj in ka Gakkai Zasshi, 38 : 1111-1114 (1986) (in Japanese)

3) Cuesta RS, Eichhorn JH, Rice LW, Fuller AF Jr, Nikrui N and Goff BA: Histologic transformation of benign endometriosis to early epithelial ovarian cancer. Gynecol Oncol 60 : 238-244 (1996)

4) Chew S, Tham KF and Ratnam SS: A series of ovarian clear cell and endometrioid carcinoma and their association with endometriosis. Singapore Med J 138 : 289-291 (1997)

5) Cancer Committee of the International Federation of Gynecology and Obstetrics: Staging announcement : FIGO Cancer Committee. Gynecol Oncol 25 : 383-385 (1986)

6) Kabawat S, Bast RC, Weich WR, Knapp RC and Colvin RB : Immunopathologic characterisation of a monoclonal antibody that recognises common surface antigens of human ovarian tumors of serous, endometroid, and clear cell type. Am J Clin Pathol 79 : 98-103 (1983)

7) Bast RC Jr, Feeney M, Lazarus H, Nadler LM, Colvin RB and Knapp BC: Reactivity of a monoclonal antibody with human ovarian carcinoma. J Clin Invest 68 : 1331-1337 (1981)

8) MacDonald F, Bird R, Stokes H, Russell B and Crocker J : The expression of CEA, CA125, CA19-9, and human milk fat globule antigen in tumors of the ovary. J Clin Pathol 41 : 260-264 (1988)

9) Shimizu Y, Kamoi S, Amada S, Akiyama F and Silverberg SG: A possible new grading system for ovarian carcinoma. Sanfujinnka Chiryou $74: 154-164$ (1997) (in Japanese)

10) Serov SF, Scully RE and Sobin LH: Histological typing of ovarian tumors. International Histological Classification of Tumors. Number 9, World Health Organization, Geneva, pp 17-54 (1973)

11) Fisher RA : On the mathematical foundation of theoretical statistics. Philos Trans $R$ Soc Lond A 222 : 309-368 (1922)

12) Kaplan E and Meier P : Nonparametric estimation for incomplete observations. J Am Stat Assoc 53 : 457-481 (1958)

13) Wilcoxon F : Individual comparisons by ranking methods. Biometrics $1: 80-83$ (1945) 
14) Kennedy AW, Biscotti CV, Hart WR and Webster KD: Ovarian clear cell adenocarcinoma. Gynecol Oncol 32 : 342-349 (1989)

15) Nakano T, Enoki K, Nakashima M, Ishikawa H, Ametani $Y$, Ohta S, Ohkuchi A, Satake S, Kojima $Y$, Funamoto $\mathbf{H}$, Tateno $M$ and Miwa A : Survival in patients with clear cell carcinoma of the ovary. Gan To Kagakurhyouhou (Jpn J Cancer Chemother) $25: 67-73$ (1998) (in Japanese)

16) Gadducci A, Ciancia EM, Campani D, Malagnino G, Luca FD, Facchini V, Pingitore R and Fioretti P: Immunohistochemical detection of p185 product, p21 product, and proliferating cell nuclear antigen (PCNA) in formalin-fixed, paraffin-embedded tissues from ovarian carcinomas : preliminary data. Eur J Gynaecol Oncol 15 : 359-368 (1994)

17) Thomas H, Nasim MM, Sarraf CE, Alison MR, Love S, Lambert HE and Price P: Proliferating cell nuclear antigen (PCNA) immunostaining : a prognostic factor in ovarian cancer ? Br J Cancer 71 : 357-362 (1995)

18) Dawson AE, Norton JA and Weinberg DS : Comparative assessment of proliferation and DNA content in breast carcinoma by image analysis and flow cytometry. Am J Pathol 136 : 1115-1124 (1990)

19) van Dierendock JH, Wijsman JH, Keijzer R, van de Velde CJH and Cornelisse CJ : Cell-cycle-related staining patterns of antiproliferating cell nuclear antigen monoclonal antibodies. Comparison with Brd Urd labeling and Ki-67 staining. Am J Pathol 138 : 1165-1172 (1991)

20) Gee JM, Douglas-Jones A, Hepburn P, Sharma AK, McClelland RA, Ellis IO and Nicholson RI : A cautionary note regarding the application of $\mathrm{Ki}-67$ antibodies to paraffin-embedded breast cancer. $J$ Pathol $177: 285-293$ (1995)

21) Hepburn P, Glynne-Jones E, Goddard L, Gee JMW and Happer ME : Cell proliferation in prostatic carcinoma : comparative analysis of Ki-67, MIB-1, and PCNA. Histochem J 27 : 196-203 (1995)

22) Tannapfel A, Hahn HA, Katalinic A, Fietkau RJ, Kuhn R and Wittekind CW : Prognostic value of ploidy and proliferation markers in renal cell carcinoma. Cancer $77: 164-171$ (1996)

23) Leonardi E, Girlando S, Serio G, Mauri FA, Perrone G, Scampini S, Dalla Palma P and Barbareschi M: PCNA and Ki-67 expression in breast carcinoma : correlations with clinical and bioligical variables. $J$ Clin Pathol 45 : 416-419 (1992)

24) Rosa JC, Mendes R, Filipe MI and Morris RW : Measurement of cell proliferation in gastric carcinoma : comparative analysis of $\mathrm{Ki}-67$ and proliferative cell nuclear antigen (PCNA). Histochem J 24 : 93-101 (1992)

25) Hall PA, Leviso DA, Woods AL, Yu CC, Kellock DB, Watkins JA, Barnes DM, Gillett CE, Camplejohn R, Dover R, Wasseem NH and Lane DP: Proliferating cell nuclear antigen (PCNA) immunolocalization in paraffin sections : an index of cell proliferation with evidence of deregulated expression in some neoplasms. $J$ Pathol 162 : 285-294 (1990)

26) Adachi S, Noda T, Kiyozuka Y, Ito K, Itani Y, Tsujimoto A, Sakakura S, Yoshida T, Umekage H, Nakayama $\mathbf{M}$ and Fujita $\mathbf{M}$ : The importance of CA125 immunohistochemical staining in patients with ovarian cancer. Nippon Sanka Fujinka Gakkai Zasshi 46 : 896-902 (1994) (in Japanese)

27) Goldie JH and Coldman AJ : A mathematical model for relating the drug sensitivity of tumors to their spontaneous mutation rate. Cancer Treat Rep 63 : 1727-1732 (1979)

[Received November 25, 1999 : Accepted December 14, 1999] 